\title{
XAISec - EXPLAINABLE AI SECURITY: AN EARLY Discussion PAPER ON NEW MULTIDISCIPLINARY SubFiEld In Pursuit of BuIlding TRUst in SECURITY OF AI SYSTEMS
}

\author{
A PREPRINT \\ ๑ Manojkumar Parmar \\ Robert Bosch Engineerıng and Business Solutions Private Limited \\ Bengaluru 560095, India \\ manojkumar . parmar@bosch.com
}

December 23, 2021

\begin{abstract}
Today's Artificial Intelligence systems are at the epicenter of security threats across industries. Attackers are trying to turn AI systems against the organization and society, intending to cause harm at various levels. Security, along with the explainability of AI, is a cornerstone for Digital Trust and Trustworthy AI. AI-based systems provide new attack surfaces, and adversaries can utilize attack surfaces to construct attacks to exploit vulnerabilities. Mission-critical systems using AI need to address the crucial problem of AI Security(AISec) and Explainable AI (XAI). In the early version of the discussion paper, we propose the new sub-field of Explainable AI Security (XAISec) at the intersection of AISec, XAI, and Explainable Security (XSec) for Mission-critical systems. We propose that XAISec should aim to explain AI Security's workings (justification of attack and transparency about defense) at an appropriate level considering multiple aspects. XAISec is a niche multidisciplinary greenfield with an ascertained need and validated using informal interview settings. We invite constructive criticism, collaboration, and contribution to jump-start the sub-field. We believe that with XAISec as an integral part of AI, AI can impact millions of lives across the globe, enabling smarter, sustainable, and evolutionary transformations.
\end{abstract}

\section{Overview}

Artificial Intelligence systems of today are at the epicenter of security threats across industries. Attackers are trying to turn AI systems against the organization and society, intending to cause harm at various levels [Par21]. With the increasing adoption of AI systems at the enterprise level, for value creation, protecting these systems throughout their life-cycle and creating a trust for AI amongst users and regulators has become the top priority for all of us.

AI-based systems provide new attack surfaces, and adversaries can utilize attack surfaces to construct attacks to exploit vulnerabilities. Recent studies showed that AI models are vulnerable to various attacks which can compromise products, services, and systems. [SSP$\left.{ }^{+} 17, \mathrm{BKA}^{+} 21\right]$ describes that Mission-critical systems using AI need to address the crucial problem of AI Security(AISec). The mission-critical systems using AI also have an additional critical challenge of explainability as per $\left[\mathrm{SSP}^{+}\right.$17]. Explainable $\mathrm{AI}(\mathrm{XAI})$ provides an appropriate level of rationale for the decision made by AI systems so that humans can understand the decision process of AI [MZR20, XUD ${ }^{+} 19$, FLV20]. Explainability is the critical aspect of building trust along with security [Shi21]. The explainability problem is not limited to the AI system but also a dire need in the security domain. [VM20] described a unique approach of Explainable Security (XSec) to address the same. 


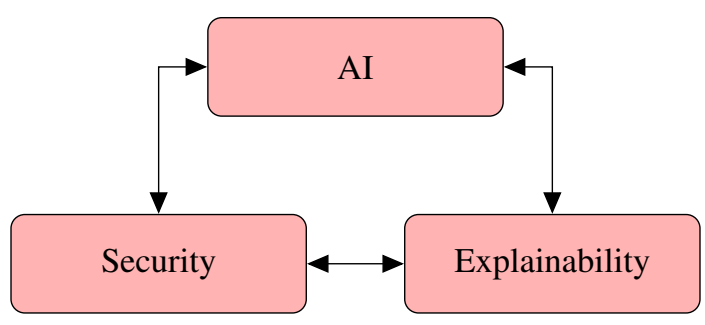

Figure 1: Intersection between fields

The author proposes the new sub-field of Explainable AI Security (XAISec) at the intersection of AI security (AISec), Explainable AI (XAI), and Explainable Security (XSec) for Mission-critical systems. The XAISec aims to provide explanations about the workings of AI Security. The explanations mainly focus on the justification of security attacks and transparency about defenses against security attacks on AI Systems [Pie11, YXZ ${ }^{+}$19]. It follows the approach of Know your model (KYM) from [RNHS21], know your attacks (KYA), and know your defenses (KYD) at the appropriate level. XAISec aims to build and reinforce trust in the AI ecosystem by holistically providing answers about security aspects of AI systems related to the AI/ML model, software, hardware, infrastructure, systems, and organization. Understanding emerging attacks and remediation of systems at the human level will help organizations stay one step ahead of malicious attackers.

\section{Proposal for Explanations}

The explanations that XAISec needs to provide vary based on detailing and aspects of explanation.

\subsection{Levels of Details}

XAISec should aim to provide explanations at mainly two levels [Pie11].

- Low Level of Detail: The low level of details explaining "why." It aims to provide justification and instill confidence in the general audience. Here are a few example questions.

- Why the AI model is vulnerable?

- Why the AI model is vulnerable to specific attacks?

- Why the AI model defenses are working?

- High Level of Detail: The experts need a high level of details explaining "how" to instill trust and transparency. Here are a few example questions.

- How are the attacks working against AI models?

- How are the defenses working against AI models?

The levels of details are highly contextualised to the model, dataset, attack surface used, and attack vectors used.

\subsection{Aspects of Explanation}

While providing explanations at multiple levels, XAISec should also consider the following aspects. [VM20].

- Why do explanations related to AISec is needed?

- Who provides the explanation about AISec?

- What is the form of explanation?

- Who receives the explanation about AISec?

- What is the end goal of delivering an explanation?

- What is explained?

- What are the details of the explanation?

- How is it explained? 
- What trade-off is needed for an explanation?

- Where is the explanation delivered?

- When is it delivered?

- How is the explanation delivered?

Considering multiple aspects will make the explanation useful, impactful, and meaningful for all stakeholders.

\section{Brief on Informal Methodology}

The author conducted five informal interviews with experts from the fields of AISec, and XAI with researchers, developers, and business owners. The interviews indicate the need for a solution for stated problems, specifically for the mission-critical system to improve reliability and liability issues of AIOT Product. In specific cases, interviewees raised concerns about destroying brand reputation, differentiation, and value proposition.

Recent academic trends in explaining adversarial attacks are the XAISec field's adjacency. However, the preliminary literature review indicates that XAISec is a new green sub-field.

\subsection{Limitation and Future work}

The discussion paper is an early version to discuss the upcoming subfield and by no means exhaustive. The author requests experts to exercise discretion about the subfield. The future work includes a detailed study to develop a comprehensive view of the subfield and list out challenges and a roadmap.

\section{Impact and Call to Action}

XAISec is a niche multidisciplinary field and has many research challenges and possible directions. XAISec field will need experts from research, academia, and industry to deliberate, discuss, understand, refine and propose a joint road map. The improvement in the XAISec field will have a distinct and exponential impact on making AI trustworthy for all [GAB ${ }^{+} 21$, Rya20, Jha19, SMN19].

Trustworthy AI is a cornerstone for realizing its transformative potential. For masses to embrace AI, security and privacy will be the first factors to be questioned. The author believes that with XAISec as an integral part of AI, AI can impact millions of lives across the globe, enabling smarter, sustainable, and evolutionary transformations.

\section{References}

$\left[\mathrm{BKA}^{+} 21\right]$ Elisa Bertino, Murat Kantarcioglu, Cuneyt Gurcan Akcora, Sagar Samtani, Sudip Mittal, and Maanak Gupta. AI for Security and Security for AI. In Proceedings of the Eleventh ACM Conference on Data and Application Security and Privacy, pages 333-334, Virtual Event USA, April 2021. ACM.

[FLV20] Andrea Ferrario, Michele Loi, and Eleonora Viganò. In AI We Trust Incrementally: a Multi-layer Model of Trust to Analyze Human-Artificial Intelligence Interactions. Philosophy \& Technology, 33(3):523-539, September 2020.

$\left[\mathrm{GAB}^{+} 21\right]$ Omri Gillath, Ting Ai, Michael S. Branicky, Shawn Keshmiri, Robert B. Davison, and Ryan Spaulding. Attachment and trust in artificial intelligence. Computers in Human Behavior, 115:106607, February 2021.

[Jha19] Susmit Jha. Trust, Resilience and Interpretability of AI Models. In Majid Zamani and Damien Zufferey, editors, Numerical Software Verification, volume 11652, pages 3-25. Springer International Publishing, Cham, 2019.

[MZR20] Sina Mohseni, Niloofar Zarei, and Eric D. Ragan. A Multidisciplinary Survey and Framework for Design and Evaluation of Explainable AI Systems. arXiv:1811.11839 [cs], August 2020. arXiv: 1811.11839.

[Par21] Manojkumar Parmar. Enemy at the Gates - The untold chronicles of AI Security - ET CIO. ETCIO, September 2021.

[Pie11] Wolter Pieters. Explanation and trust: what to tell the user in security and AI? Ethics and Information Technology, 13(1):53-64, March 2011. 
[RNHS21] Mary Roszel, Robert Norvill, Jean Hilger, and Radu State. Know Your Model (KYM): Increasing Trust in AI and Machine Learning. arXiv:2106.11036 [cs], May 2021. arXiv: 2106.11036.

[Rya20] Mark Ryan. In AI We Trust: Ethics, Artificial Intelligence, and Reliability. Science and Engineering Ethics, 26(5):2749-2767, October 2020.

[Shi21] Donghee Shin. The effects of explainability and causability on perception, trust, and acceptance: Implications for explainable AI. International Journal of Human-Computer Studies, 146:102551, February 2021.

[SMN19] Shervin Shahrdar, Luiza Menezes, and Mehrdad Nojoumian. A Survey on Trust in Autonomous Systems. In Kohei Arai, Supriya Kapoor, and Rahul Bhatia, editors, Intelligent Computing, Advances in Intelligent Systems and Computing, pages 368-386, Cham, 2019. Springer International Publishing.

$\left[\mathrm{SSP}^{+}\right.$17] Ion Stoica, Dawn Song, Raluca Ada Popa, David Patterson, Michael W. Mahoney, Randy Katz, Anthony D. Joseph, Michael Jordan, Joseph M. Hellerstein, Joseph E. Gonzalez, Ken Goldberg, Ali Ghodsi, David Culler, and Pieter Abbeel. A Berkeley View of Systems Challenges for AI. arXiv:1712.05855 [cs], December 2017. arXiv: 1712.05855.

[VM20] Luca Viganò and Daniele Magazzeni. Explainable Security. In 2020 IEEE European Symposium on Security and Privacy Workshops (EuroS PW), pages 293-300, September 2020.

$\left[\mathrm{XUD}^{+}\right.$19] Feiyu Xu, Hans Uszkoreit, Yangzhou Du, Wei Fan, Dongyan Zhao, and Jun Zhu. Explainable AI: A Brief Survey on History, Research Areas, Approaches and Challenges. In Jie Tang, Min-Yen Kan, Dongyan Zhao, Sujian Li, and Hongying Zan, editors, Natural Language Processing and Chinese Computing, volume 11839, pages 563-574. Springer International Publishing, Cham, 2019.

[YXZ ${ }^{+}$19] Jirong Yi, Hui Xie, Leixin Zhou, Xiaodong Wu, Weiyu Xu, and Raghuraman Mudumbai. Trust but Verify: An Information-Theoretic Explanation for the Adversarial Fragility of Machine Learning Systems, and a General Defense against Adversarial Attacks. arXiv:1905.11381 [cs, stat], May 2019. arXiv: 1905.11381. 